\title{
Women's persistent depressive and perinatal grief symptoms following a miscarriage: the role of childlessness and satisfaction with healthcare services
}

\author{
Francine deMontigny $^{1} \cdot$ Chantal Verdon $^{1} \cdot$ Sophie Meunier $^{2} \cdot$ Diane Dubeau $^{1}$
}

Received: 8 September 2016 / Accepted: 30 May 2017 / Published online: 16 June 2017

(C) The Author(s) 2017. This article is an open access publication

\begin{abstract}
The objectives of this cross-sectional study were to determine whether depressive and perinatal grief symptoms vary according to time since miscarriage and to test whether childlessness and satisfaction with healthcare services influence symptom duration. A total of 245 women who had experienced a miscarriage answered a self-report questionnaire, indicating the date of their miscarriage and assessing their present level of depressive and perinatal grief symptoms. They also provided sociodemographic characteristics and indicated their level of satisfaction with healthcare services. One-way analyses of variance indicated that women who had miscarried within the past 6 months reported higher scores for depressive symptoms than did women who had miscarried between 7 and 12 months ago and more than 2 years ago. However, when controlling for childlessness and satisfaction with healthcare services, those differences became respectively marginal and non-significant, indicating that depressive symptoms are similar across time for more than 2 years after the loss. Regarding perinatal grief, results revealed that symptoms significantly decreased across time only for women with children and women who were satisfied with healthcare services. For childless women and those dissatisfied with healthcare services, perinatal grief symptoms did not vary according to time since miscarriage. Results suggest that, particularly for women who are childless and/or dissatisfied with healthcare services, depressive and perinatal grief symptoms persist long after a miscarriage. These results highlight the
\end{abstract}

Francine deMontigny

francine.demontigny@uqo.ca

1 Université du Québec en Outaouais, PO Box 1250, Hull Station, Gatineau, QC J8X 3X7, Canada

2 Université du Québec à Montréal, Montreal, Québec, Canada importance of paying particular attention to more vulnerable women and of improving healthcare services postmiscarriage.

Keywords Miscarriage - Depression - Perinatal grief . Childlessness $\cdot$ Satisfaction with healthcare services

\section{Introduction}

Miscarriage, defined as the premature loss of an embryo or a fetus within the first 27 weeks of pregnancy (Klier et al. 2002), is an important and prevalent event. Indeed, about $15 \%$ of all clinically recognized pregnancies end in miscarriage (Hemminki and Forssas 1999; Kline et al. 1989). Many studies have reported the deleterious impact of miscarriage on women's mental health (Beutel et al. 1995; Klier et al. 2002; Neugebauer et al. 1992; Neugebauer et al. 1997). However, results concerning the duration of depressive and perinatal grief symptoms following a miscarriage are inconsistent. Indeed, some studies have indicated that, for most women, symptoms resorb and are similar to those of the general population within 6 months to 1 year (Brier 2008; Broen et al. 2006; Janssen et al. 1996; Cumming et al. 2007; Lok et al. 2010), while others have indicated that depressive symptoms persist long after the loss, for up to 3 years (Beutel et al. 1995; Blackmore et al. 2011). These results raise the question, why do some women recover more rapidly than others from the psychological burden of a miscarriage? The aim of the present study was to provide some answers to this question by examining whether depressive and perinatal grief symptoms vary according to time since miscarriage, and whether childlessness and satisfaction with healthcare services can influence the association between depressive and perinatal grief symptoms and time since miscarriage. 


\section{Miscarriage and women's mental health}

There is increasing recognition that miscarriage can seriously affect women's mental health. In fact, miscarriage, usually seen as the death of an expected child, is considered a traumatic event (Klier et al. 2002). Empirical studies have reported elevated depressive symptoms among women in the months following a miscarriage (Beutel et al. 1995; Lok et al. 2010; Neugebauer et al. 1992). Moreover, studies have found that 10 to $15 \%$ of women who experience a miscarriage attain the clinical threshold for a major depressive disorder in the months following the event (Beutel et al. 1995; Cumming et al. 2007; Neugebauer et al. 1997).

In addition to depressive symptoms, after a miscarriage, women may experience perinatal grief and have symptoms of bereavement, such as emotional numbness and a yearning for the lost child (Beutel et al. 1995; Lee and Rowlands 2015). Indeed, miscarriage is comparable to other forms of loss in terms of intensity and duration (Brier 2008). Even though grief and depression share some similarities, they can be assessed and experienced separately. As pointed out by Lee and Rowlands (2015), following a miscarriage, some women may report grief symptoms, such as struggling with difficult emotions and trying to find meaning, while at the same time not experiencing depressive symptoms and being able to function well enough in everyday life. To date, however, most studies on miscarriage have focused on symptoms of anxiety and depression, and few have examined both depression and perinatal grief at the same time (see Beutel et al. 1995 for an exception).

\section{Duration of depressive and perinatal grief symptoms following a miscarriage}

Results concerning the duration of depressive and perinatal grief symptoms following a miscarriage are inconsistent. Some studies have found that, for most women, depressive and perinatal grief symptoms resorb within 6 months to 1 year following the miscarriage (Brier 2008; Broen et al. 2006; Janssen et al. 1996; Cumming et al. 2007; Lok et al. 2010), while others have found that the deleterious effects of miscarriage on women's mental health persist long after the loss. For example, Blackmore et al. (2011) found that depression and anxiety could persist up to 3 years after the miscarriage. In the same vein, Beutel et al. (1995) found that, while grief symptoms appear to last around 6 months, anxious and depressive symptoms persisted more than 1 year past the loss. Those results partly contradict what Lee and Rowlands (2015) found when integrating the results of both quantitative and qualitative studies, which was that some women might not experience long-lasting depressive symptoms yet still grieve for their loss many years following a miscarriage. Thus, results concerning the duration of depressive and perinatal grief symptoms following a miscarriage are inconsistent. Also, very few studies have exceeded the 1-year time frame usually applied in longitudinal studies examining the association between miscarriage and mental health, and little is known about its long-term impact.

Moderating variables have been identified to explain the inconsistent results concerning the association between mental health and time since miscarriage. For example, Lok et al. (2010) found that depressive symptoms persist 1 year after the loss only among women who were initially more distressed. Rowlands and Lee (2010a) also found that previous medically diagnosed depression or anxiety was associated with downward mental health trajectories across time among women who had miscarried. In addition to prior mental health, childlessness and satisfaction with healthcare services could also be important to consider when explaining why depressive and perinatal grief symptoms persist longer after a miscarriage for some women.

Childlessness has consistently been associated with depressive and perinatal grief symptoms after a miscarriage (Janssen et al. 1997; Neugebauer et al. 1992, 1997). Indeed, women with one or more living children have been found to be less at risk of developing depression (Neugebauer et al. 1997) or perinatal grief symptoms (Janssen et al. 1997) following a miscarriage. Along those lines, studies have examined whether a subsequent pregnancy and the birth of a healthy child might reduce depressive and grief symptoms related to a prior miscarriage. Blackmore et al. (2011) found that, among women with prior prenatal loss, depressive symptoms during a subsequent pregnancy persisted even after the birth of a healthy child. However, other studies have reported that a subsequent pregnancy is associated with a significant decrease in grief level (Cuisinier et al. 1996; Nikcevic et al. 1999). Brier (2008) suggests that a subsequent pregnancy enables women to retrieve important roles they had lost with the miscarriage (e.g., role of pregnant woman and mother). Retrieving those roles might help them to recover more rapidly from the grief associated with their miscarriage. Based on the inconsistent results mentioned above, it is conceivable that the presence of living children might influence perinatal grief more than depressive symptoms, but this hypothesis would need to be tested.

Regarding satisfaction with healthcare services, studies have indicated that support from health professionals has a positive impact on women's experience of a miscarriage (Rowlands and Lee 2010b) and that women who were satisfied with their physician reported higher mental health scores (Rowlands and Lee 2010a). Despite the recognized importance of quality of health care following a miscarriage, studies continue to report that a majority of women are dissatisfied with the care they received (Rowlands and Lee 2010b; Simmons et al. 2006; Warner et al. 2012). Lack of empathy, 
compassion, information, and follow-up are identified as dissatisfaction factors (Geller et al. 2010). Further research is needed on the influence of satisfaction with healthcare services on women's mental health following a miscarriage, including a variety of health services (e.g., nurses, midwives, psychologists), and on how satisfaction levels might explain the greater persistence of depressive and grief symptoms among some women.

\section{Study objectives}

Results from previous studies highlight the need to examine the duration of depressive and perinatal grief symptoms following a miscarriage and to identify what factors might help some women recover faster than others from this experience. The first objective of the present study was to examine the level of depressive and perinatal grief symptoms following a miscarriage according to time since miscarriage. To add to existing studies, time since miscarriage was extended to include more than 2 years after the loss. The second objective was to examine whether the presence of children and satisfaction with healthcare services can influence the association between time since miscarriage and depressive and perinatal grief symptoms. The aim was to gain a better understanding of the conditions under which perinatal grief and depressive symptoms might resorb faster and to identify areas of vulnerability where attention is needed to mitigate the deleterious effect of miscarriage on women's mental health.

\section{Methods}

\section{Procedure and participants}

A cross-sectional study was conducted in the province of Quebec, Canada. Women were recruited through ads posted on conventional and social media. Ads were also placed in medical clinics and emergency rooms and conveyed in bereavement support groups, and participants were referred from health professionals working in those settings. Women interested in participating called a research assistant, who verified whether they met the inclusion criteria: (1) aged 18 years and older; (2) having experienced at least one miscarriage in the past 6 years; and (3) being able to read French. Eligible participants could answer the selfreport questionnaire either by phone with the research assistant, or on their own, on a secured web platform. A total of 245 women answered the questionnaire, all of whom chose to use the online version.

\section{Measures}

Depression The French version of the Edinburgh Postnatal Depression Scale (Cox et al. 1987; Guedeney and Fermanian 1998) was used to assess depressive symptoms. This ten-item scale shows good face, criterion, and construct validity (Guedeney and Fermanian 1998). Its reliability has also been demonstrated with satisfactory internal consistency $(\alpha=0.76)$ and high test-retest reliability $(\mathrm{ICC}=0.91)$ (Guedeney and Fermanian 1998). Respondents are asked to indicate how they have felt over the past 7 days, using a 4point Likert scale ranging from 0 to 3 . Scores for all the questions are summed to obtain a global score ranging from 0 to 30 ( $\alpha=0.89$ in the present study). A score of 10 or higher is usually used as a cutoff for possible depression (Cox et al. 1987).

Perinatal grief The French version of the Perinatal Grief Scale-Short Version (Potvin et al. 1989; de Tychey and Dollander 2000) was used to measure perinatal grief symptoms. This 33-item scale shows good psychometric characteristics (de Tychey and Dollander 2000). Respondents are asked to indicate how they presently feel regarding their miscarriage (e.g., I am grieving for the baby). Reversed scores were recoded, and items were summed to produce a global perinatal grief score ( $\alpha=0.94$ in the present study). A score $>91$ is considered high (Toedter et al. 2001).

Satisfaction with healthcare services A 19-item scale was created to assess women's satisfaction with healthcare services. Participants were asked to indicate how helpful they perceived a list of 19 professional health services received during their miscarriage (nurse, physician, gynecologist) or afterward (support groups) on a 4-point Likert scale $(1=$ not helpful; 2 = somewhat helpful; $3=$ helpful; 4 = very helpful). Participants could also indicate "not applicable" if they had not received the service during their miscarriage or afterward. Scores for each service received were averaged to obtain a global satisfaction score. Participants with a mean score $\geq 3$ were classified as satisfied, with the remainder classified as dissatisfied.

Sociodemographic variables and childlessness Participants were asked to indicate their age, household income, highest education level, and country of birth (Canada/other). They were also asked to indicate the date of their latest miscarriage. Time since miscarriage was categorized as less than 7 months, between 7 and 12 months, between 1 and 2 years, and more than 2 years. Participants were asked to indicate the number of living children they presently had. A dichotomous variable was created: $1=$ childless and $0=$ with children. 


\section{Statistical analysis}

First, missing data were calculated. Since they were minimal (less than 1\%), nothing was done to replace them and listwise deletion was used for main analysis. Then, descriptive statistics were obtained. The number of participants in each category was calculated for categorical variables, while mean and standard deviation were calculated for depression and perinatal grief continuous scores. One-way analyses of variance (ANOVA) were conducted to examine whether depressive and perinatal grief symptoms varied by time since miscarriage. When a significant result was found $(p<.05)$, Tukey's post hoc tests were performed to investigate where the difference was positioned in relation to the time points. Finally, to test the influence of childlessness and satisfaction with healthcare services on the association between depressive and perinatal grief symptoms and time since miscarriage, 2 (childlessness or satisfaction with healthcare services) $\times 4$ (time since miscarriage) ANOVAs were performed. When a significant interaction was found, file splitting was used and ANOVAs were performed to examine whether depressive and perinatal grief symptoms varied according to time since miscarriage at different levels of childlessness and/or satisfaction with healthcare services. To control for family-wise alpha error rate while testing for those two simple effects for each analysis, statistical significance was lowered to $p<.025$.

\section{Results}

Table 1 presents participants' characteristics. Mean age was 31 years, with a majority of participants between 25 and 34 years. Most participants were born in Canada, more than three quarters had a household income of at least 50,000 CAD and most had a college (technical or pre-university) or university degree. Participants were equally distributed by time since miscarriage, with about one quarter of the sample in each category. Participants in each of those categories did not differ significantly in terms of sociodemographic characteristics (age, education, income, country of birth). Finally, more than one third of participants were childless and almost one third were dissatisfied with the healthcare services they received.

Table 2 presents mean scores for depression and perinatal grief symptoms by time since miscarriage. One-way ANOVAs indicated significant differences in depressive symptoms $\left(F_{(3,241)}=3.24, p=.023\right)$ according to time since miscarriage. More specifically, Tukey's post hoc tests indicated that women who had miscarried in the past 6 months had a higher score for depressive symptoms than did women who had miscarried between 7 and 12 months ago or more than 2 years ago. However, the score for depressive symptoms among women who had miscarried between 1 and 2 years ago was similar to that of women who had miscarried within
Table 1 Participants' characteristics

Number $(\%)^{\mathrm{a}}$

\begin{tabular}{|c|c|}
\hline \multicolumn{2}{|l|}{ Age (years) } \\
\hline $18-24$ & $21(8.57)$ \\
\hline $25-34$ & $167(68.16)$ \\
\hline$\geq 35$ & $55(22.45)$ \\
\hline \multicolumn{2}{|l|}{ Family income (CAD) } \\
\hline $0-49,999$ & $54(22.04)$ \\
\hline $50,000-99,999$ & $108(44.08)$ \\
\hline$\geq 100,000$ & $83(33.88)$ \\
\hline \multicolumn{2}{|l|}{ Education } \\
\hline High school & $39(15.92)$ \\
\hline College (technical or pre-university) & $72(29.39)$ \\
\hline University & $130(53.06)$ \\
\hline \multicolumn{2}{|l|}{ Country of birth } \\
\hline Outside of Canada & $17(6.94)$ \\
\hline Canada & $228(93.06)$ \\
\hline \multicolumn{2}{|l|}{ Time since miscarriage } \\
\hline $0-6$ months & $65(26.53)$ \\
\hline $7-12$ months & $58(23.67)$ \\
\hline $1-2$ years & $64(26.51)$ \\
\hline$>2$ years & $58(23.67)$ \\
\hline \multicolumn{2}{|l|}{ Childlessness } \\
\hline Yes & $92(37.55)$ \\
\hline No & $153(62.45)$ \\
\hline \multicolumn{2}{|l|}{ Satisfaction with healthcare services } \\
\hline Satisfied & $160(65.31)$ \\
\hline Dissatisfied & $78(31.84)$ \\
\hline
\end{tabular}

${ }^{a}$ Percentage ratios are based on the total $N(245)$

the past 6 months. As for perinatal grief, results indicated no significant difference in mean score according to time since miscarriage $\left(F_{(3,241)}=2.49, p=.061\right)$.

To examine the influence of childlessness on the association between time since miscarriage and women's depressive and perinatal grief symptoms, 4 (time since miscarriage) $\times 2$ (childlessness yes/no) ANOVAs were conducted. Regarding depressive symptoms, results revealed that the main effect of childlessness $\left(F_{(1,237)}=8.60, p=.004\right)$ was significant. Childless women had higher scores for depressive symptoms than did women who had children ( $M=12.24$ vs. 9.88). The main effect of time since miscarriage was marginally significant $\left(F_{(3,237)}=2.63, p=.051\right)$ while the interaction between time since miscarriage and childlessness $\left(F_{(3,237)}=2.22\right.$, $p=.087$ ) was not.

Similarly, for perinatal grief, the main effect of childlessness was significant $\left(F_{(1,237)}=31.00, p<.001\right)$, while the main effect of time since miscarriage was not $\left(F_{(3,237)}=1.28\right.$, $p=.282)$. Childless women had a higher score for perinatal grief symptoms ( $M=84.38$ vs. 64.09$)$ than did women with 
Table 2 Depression and perinatal grief symptoms by time since miscarriage

\begin{tabular}{|c|c|c|c|c|c|c|}
\hline & \multicolumn{4}{|c|}{ Time since miscarriage } & \multirow[t]{2}{*}{$F$} & \multirow[t]{2}{*}{$p$} \\
\hline & $0-6$ months & $7-12$ months & $1-2$ years & $>2$ years & & \\
\hline Depressive symptoms & $12.62(5.67)$ & $9.66(6.51)$ & $10.97(6.37)$ & $9.57(6.45)$ & 3.24 & .02 \\
\hline $\begin{array}{l}\text { Mean (SD) } \\
\text { Perinatal grief symptoms } \\
\text { Mean (SD) }\end{array}$ & $78.49(26.60)$ & $68.88(30.22)$ & $73.45(31.90)$ & $65.02(26.48)$ & 2.49 & .06 \\
\hline
\end{tabular}

children. The interaction between time since miscarriage and childlessness was significant $\left(F_{(3,237)}=2.68, p=.047\right)$. As illustrated in Fig. 1, for women with children, perinatal grief symptoms decreased by time since miscarriage, while for childless women, perinatal grief symptoms increased and remained stable. Indeed, decomposition of main effects revealed that, for childless women, perinatal grief levels did not vary according to time since miscarriage $\left(F_{(3,88)}=1.11\right.$, $p=.348)$. However, there was a significant difference among women with children $\left(F_{(3,149)}=3.70, p=.013\right)$, where women who had miscarried more than 2 years ago $(M=75.32$, $\mathrm{SD}=26.33$ ) reported significantly fewer perinatal grief symptoms than did women who had miscarried within the past 6 months $(M=58.54, \mathrm{SD}=22.11)$.

Finally, to examine the influence of satisfaction with healthcare services on the association between time since miscarriage and women's depressive and perinatal grief symptoms, 4 (time since miscarriage) $\times 2$ (satisfaction with healthcare services yes/no) ANOVAs were conducted. Results revealed that, for depressive symptoms, neither the main effects of time since miscarriage $\left(F_{(3,230)}=2.22\right.$, $p=.086)$ and of satisfaction with healthcare services $\left(F_{(1,230)}=3.05, p=.082\right)$ nor the interaction of those two variables $\left(F_{(3,230)}=2.01, p=.114\right)$ were significant. As for perinatal grief, the main effect of time since miscarriage was not significant when included with satisfaction with care in the eq. $\left(F_{(3,230)}=1.59, p=.193\right)$, while the main effect of satisfaction with care was significant $\left(F_{(1,230)}=7.32, p=.007\right)$. Women who were satisfied with healthcare services had lower scores for perinatal grief symptoms $(M=67.75, \mathrm{SD}=26.14)$ than did women who were not $(M=77.88, \mathrm{SD}=31.74)$. The interaction between time since miscarriage and satisfaction with healthcare services was marginally significant $\left(F_{(3,230)}=2.63, p=.051\right)$. As illustrated in Fig. 2, among women who were satisfied with healthcare services, perinatal grief symptoms decreased with time. In contrast, among women who were dissatisfied with healthcare services, there was an increase in perinatal grief symptoms for those who had miscarried between 1 and 2 years ago. Decomposition of main effects revealed that, for women who were dissatisfied with healthcare services, there was no difference in perinatal grief symptoms according to time since miscarriage $\left(F_{(3,74)}=1.35, p=.265\right)$. However, there was a significant difference among women who were satisfied with healthcare services $\left(F_{(3,156)}=3.31, p=.022\right)$. Specifically, there was a significant difference between women who had miscarried more than 2 years ago $(M=61.28 \mathrm{SD}=20.28)$ and women who had miscarried in the past 6 months $(M=78.00 \mathrm{SD}=26.43)$.

\section{Discussion}

The purpose of the present study was to examine postmiscarriage depressive and perinatal grief symptoms according to time since miscarriage. Results indicated higher scores of depressive symptoms among women who had recently miscarried, with those who had miscarried within the past 6 months having higher scores for depressive symptoms than those who had miscarried between 7 and 12 months ago or more than 2 years ago. At first glance, these results are in line with those of other studies showing a significant reduction of depressive symptoms between 6 months and 1 year after miscarriage (Brier 2008; Broen et al. 2006; Cumming et al. 2007; Janssen et al. 1996; Lok et al. 2010). However, over the longer term, it can be seen that women who had miscarried between 1 and 2 years ago continued to report high levels of depressive symptoms and did not significantly differ from women who had miscarried within the past 6 months. Hence, while depressive symptoms scores were lower among women who had miscarried between 7 and 12 months ago, there was an

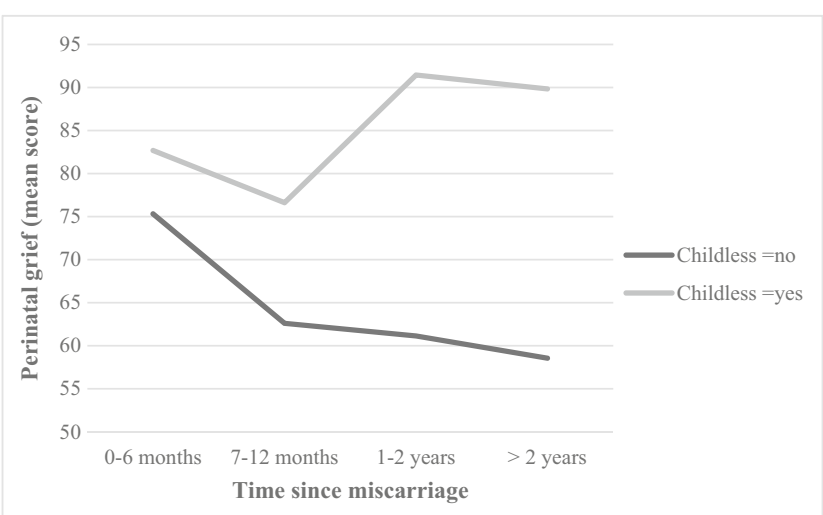

Fig. 1 Perinatal grief symptoms as a function of time since miscarriage and of childlessness 


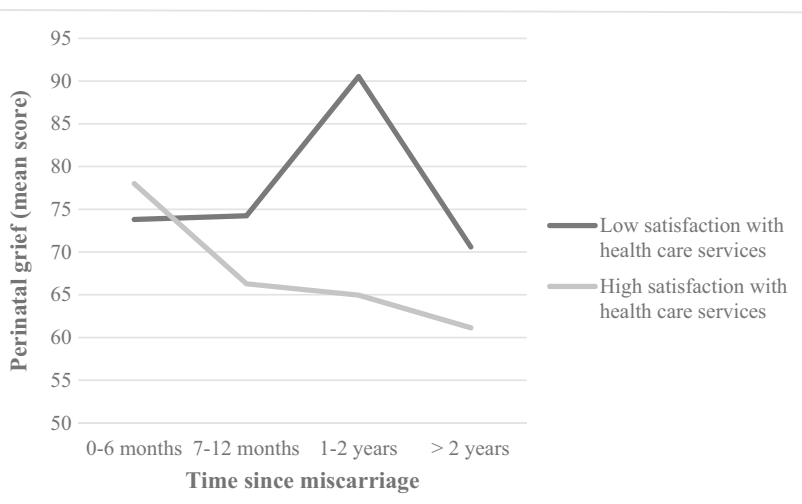

Fig. 2 Perinatal grief score as function of time since miscarriage and satisfaction with healthcare services

apparent upsurge in symptoms among women who had miscarried between 1 and 2 years ago. Difficulties conceiving another child, anniversaries, or other significant dates associated with the miscarriage might explain this upsurge in depressive symptoms. However, further analysis indicated that when controlling for childlessness and satisfaction with healthcare services, the effect of time since miscarriage on depressive symptoms became respectively marginally and non-significant, indicating that those symptoms remain stable, even long after the miscarriage. Also, with regard to perinatal grief, there was no significant reduction in symptoms for up to more than 2 years after the miscarriage. In sum, the results of the present study converge with those of Blackmore et al. (2011) and, to some extent, of Beutel et al. (1995) in finding that depressive and perinatal grief symptoms can persist long after the loss, for up to 3 years past the miscarriage.

The second objective of the study was to identify factors that might explain why depressive and perinatal grief symptoms might persist longer after a miscarriage. Childlessness was identified as one such factor. Indeed, for childless women, there was no significant difference in perinatal grief symptoms according to time since miscarriage. For those women, grief symptoms remained stable regardless of time since miscarriage, and those who had miscarried more than 2 years ago showed levels of perinatal grief similar to those of women who had recently miscarried. In contrast, for women with children, perinatal grief symptoms were significantly lower among women who had miscarried more than 2 years ago. These results are in line with those of other studies reporting that women with living children are at lower risk of depression following a miscarriage (Janssen et al. 1997; Neugebauer et al. 1997) and that grief symptoms decreased after the subsequent birth of a healthy child (Cuisinier et al. 1996; Nikcevic et al. 1999). However, it was not possible in the present study to determine whether the children were born before or after the miscarriage. Future studies should try to distinguish between those two situations and discern their respective impacts on depressive and perinatal grief symptoms. Nevertheless, childless women appear to be more vulnerable to the persistence of grief symptoms long after a miscarriage. In a randomized control trial evaluating the efficacy of a supportive counseling program for women who had miscarried, Kong et al. (2014) found that not all women benefited from the program and noted that women who were more vulnerable (i.e., women with high levels of psychological distress) should be targeted. In this vein, results of the present study indicate that health professionals should pay particular attention to childless women and follow up with them on a long-term basis.

Nonetheless, it should be noted that in the present study, childlessness did not influence the association between depressive symptoms and time since miscarriage. This result is similar to that of Blackmore et al. (2011), who found that, among women who had miscarried, depressive symptoms remained stable after the subsequent birth of a healthy child. Thus, childlessness appears to be more useful to explain the duration of perinatal grief rather than depressive symptoms.

Satisfaction with healthcare services also emerged as an important factor in the association between perinatal grief symptoms and time since miscarriage. For women who were dissatisfied with their healthcare services, there were no differences in perinatal grief symptoms across time: women who had miscarried more than 2 years ago showed levels of perinatal grief symptoms similar to those of women who had miscarried more recently. In contrast, for women who were satisfied with the healthcare services they received, levels of perinatal grief symptoms decreased by time since miscarriage and were significantly lower among women who had miscarried more than 2 years ago. These results strongly suggest that the quality of healthcare services for women going through a miscarriage might directly influence their mental health in the following months and even years. It should be noted, however, that the measure of satisfaction with healthcare services was rather heterogeneous, with participants only assessing healthcare services they received during and after their miscarriage (indicating not applicable for other services). It is therefore not possible to identify which health services (i.e., nurses, physicians, support groups, etc.) are more likely to contribute to women's satisfaction. Future studies could assess satisfaction with healthcare more precisely by focusing on one type of service at a time. Nevertheless, studies to date have reported that women are generally mostly dissatisfied with the care they received before, during, and after their miscarriage (Rowlands and Lee 2010b; Simmons et al. 2006; Warner et al. 2012), indicating that there is much to be done to assess women's needs and tailor healthcare services accordingly. Early pregnancy assessment clinics (EPAC), successfully implemented since 1991 in the UK and, more recently, in Australia, New Zealand, and Canada (Tunde-Byass and Cheung 2009; Rhone et al. 2012), appear to be an innovative solution to improve women's satisfaction with services. In an 
EPAC, specialized personnel (physician and nurse) support women in the natural, medical, or surgical resolution of their miscarriage. An evaluation conducted in Australia showed that EPAC clinics reduced emergency wait times by $55 \%$, repeat visits for the same problem by $48 \%$, and request for laboratory tests by 56\% (O'Rourke and Wood 2009). Evaluations of Canadian EPAC clinics have obtained similar results, also confirming client satisfaction (Tunde-Byass and Cheung 2009; Rhone et al. 2012). This care model is thus interesting in terms of improving quality of care and reducing health system costs and should be considered for wider deployment.

Finally, as with childlessness, satisfaction with healthcare services did not influence the association between time since miscarriage and depressive symptoms. Future studies should focus on other variables that could explain under what circumstances depressive symptoms are more likely to resorb faster. For instance, previous history of depression is strongly associated with postpartum depression (Neugebauer et al. 1997) and is probably an important variable to consider. However, it was not assessed in the present study. The use of a more precise measure of depression, such as the Structured Clinical Interview for DSM IV (SCID interview), could have given important information about the history of depression (e.g., number of episodes, chronicity) and would be recommended for future studies. Another limitation of the present study concerns the cross-sectional design, which precludes any cause-and-effect conclusions. Also, since a convenience sample was used, it is possible that women with higher levels of depressive and perinatal grief symptoms following their miscarriage were more drawn to the present study and volunteered to participate, even if their miscarriage had happened a long time ago. Notwithstanding this recruitment bias, the results of the present study are consistent with those of studies with large numbers of participants (Blackmore et al. 2011; Rowlands and Lee 2010a, 2010b). Finally, it should be noted that the question assessing childlessness did not specify whether the other children, if any, were born before or after the miscarriage (for women who had miscarried more than 1 year ago). Thus, it is not possible to know whether it was the presence of a living child before the miscarriage or the birth of a subsequent healthy child, or even both, that influenced the duration of depressive and perinatal grief symptoms.

\section{Conclusion}

Miscarriage has important repercussions on women's mental health. The present study indicates that depressive and perinatal grief symptoms can persist long after the loss. Results also revealed that perinatal grief symptoms significantly decreased across time only for women with children and women who were satisfied with healthcare services. For childless women and those dissatisfied with healthcare services, perinatal grief symptoms did not vary according to time since miscarriage and were stable for more than 2 years after the loss. Those results were not replicated with depressive symptoms, since childlessness and satisfaction with healthcare services did not influence symptom reduction across time. Other relevant variables, such as mental health history, should be taken into account in future studies. Nevertheless, these results highlight the need to optimize healthcare services offered to women before, during, and after a miscarriage. They also emphasize the importance of paying particular attention to vulnerable women and offering them support on a long-term basis.

Acknowledgements The authors would like to thank their colleagues, Dr. Carl Lacharité, Dr. Eric Tchouaket, and Dr. Martin St-André, for their backing of this line of research. They would like to acknowledge the outstanding contributions of members of the Centre for Studies and Research on Family Interventions, especially Emmanuelle Dennie Fillion and Pascale deMontigny Gauthier, research coordinators, and Jici Lord Gauthier, research assistant, for their support in data collection. We also wish to thank the participating mothers for their generosity with their time in a demanding period of their life.

\section{Compliance with ethical standards}

Funding The authors declare that this research was funded by a grant from the Fonds Québécois de recherche—santé (\#26811; 2013-2017).

Conflict of interest The authors declare that they have no conflicts of interest.

Ethical approval All procedures performed in this study were in accordance with the ethical standards of the institutional research committee and with the 1964 Helsinki Declaration and its later amendments or comparable ethical standards.

Informed consent Informed consent was obtained from all individual participants included in the study.

Open Access This article is distributed under the terms of the Creative Commons Attribution 4.0 International License (http:// creativecommons.org/licenses/by/4.0/), which permits unrestricted use, distribution, and reproduction in any medium, provided you give appropriate credit to the original author(s) and the source, provide a link to the Creative Commons license, and indicate if changes were made.

\section{References}

Beutel M, Deckardt R, von Rad M, Weiner H (1995) Grief and depression after miscarriage: their separation, antecedents, and course. Psychosom Med 57:517-526

Blackmore ER, Côté-Arsenault D, Tang W, Glover V, Evans J, Golding J, O'Connor TG (2011) Previous prenatal loss as a predictor of perinatal depression and anxiety. Br J Psychiatry 198:373-378. doi:10. 1192/bjp.bp. 110.083105

Brier N (2008) Grief following miscarriage: a comprehensive review of the literature. J Women's Health 17:451-464. doi:10.1089/jwh. 2007.0505 
Broen NA, Moum T, Sejersted Bödtker A, Ekeberg Ö (2006) Predictors of anxiety and depression following pregnancy termination: a longitudinal five-year follow-up study. Acta Obstet Gynecol Scand 85: 317-323. doi:10.1080/00016340500438116

Cox JL, Holden JM, Sagovsky R (1987) Detection of postnatal depression. Development of the 10-item Edinburgh Postnatal Depression Scale. Br J Psychiatry 150:782-786

Cuisinier M, Janssen H, de Graauw C, Bakker S, Hoogduin C (1996) Pregnancy following miscarriage: course of grief and some determining factors. J Psychosom Obstet Gynaecol 17:168-174

Cumming GP, Klein S, Bolsover D, Lee AJ, Alexander DA, Maclean M, Jurgens JD (2007) The emotional burden of miscarriage for women and their partners: trajectories of anxiety and depression over 13 months. BJOG 114:1138-1145. doi:10.1111/j.1471-0528.2007. 01452.x

de Tychey C, Dollander M (2000) Présentation de l'échelle de deuil de Potvin, Lasker et Toedter: utilité en clinique pré- et périnatale. Neuropsychiatr Enfance Adolesc 48:245-252

Geller PA, Psaros C, Kornfield SL (2010) Satisfaction with pregnancy loss aftercare: are women getting what they want? Arch Womens Ment Health 13:111-124. doi:10.1007/s00737-010-0147-5

Guedeney N, Fermanian J (1998) Validation study of the French version of the Edinburgh Postnatal Depression Scale (EPDS): new results about use and psychometric properties. Eur Psychiatry 13:83-89. doi:10.1016/S0924-9338(98)80023-0

Hemminki E, Forssas E (1999) Epidemiology of miscarriage and its relation to other reproductive events in Finland. Am J Obstet Gynecol 181:396-401

Janssen HJ, Cuisinier MC, Hoogduin KA, de Graauw KP (1996) Controlled prospective study on the mental health of women following pregnancy loss. Am J Psychiatry 153:226-230

Janssen HJ, Cuisinier MC, de Graauw KP, Hoogduin KA (1997) A prospective study of risk factors predicting grief intensity following pregnancy loss. Arch Gen Psychiatry 54:56-61

Klier CM, Geller PA, Ritsher JB (2002) Affective disorders in the aftermath of miscarriage: a comprehensive review. Arch Womens Ment Health 5:129-149. doi:10.1007/s00737-002-0146-2

Kline J, Stein Z, Susser M (1989) Conception to birth: epidemiology of prenatal development. Oxford University Press, New York

Kong GWS, Chung TKH, Lok IH (2014) The impact of supportive counselling on women's psychological wellbeing after miscarriage - a randomised controlled trial. BJOG 121:1253-1262. doi: $10.1111 / 1471-0528.12908$

Lee C, Rowlands IJ (2015) When mixed methods produce mixed results: integrating disparate findings about miscarriage and women's wellbeing. Br J Health Psychol 20:36-44. doi:10.1111/bjhp.12121
Lok IH, Yip ASK, Lee DTS, Sahota D, Chung TKH (2010) A 1-year longitudinal study of psychological morbidity after miscarriage. Fertil Steril 93:1966-1975. doi:10.1016/j.fertnstert.2008.12.048

Neugebauer R, Kline J, O'Connor P, Shrout P, Johnson J, Skodol A, Wicks J, Susser M (1992) Determinants of depressive symptoms in the early weeks after miscarriage. Am J Public Health 82:13321339

Neugebauer R, Kline J, Shrout P, Skodol A, O’Connor P, Geller PA, Stein Z, Susser M (1997) Major depressive disorder in the 6 months after miscarriage. JAMA 277:383-388

Nikcevic AV, Tunkel SA, Kuczmierczyk AR, Nicolaides KH (1999) Investigation of the cause of miscarriage and its influence on women's psychological distress. BJOG 106:808-813

O'Rourke D, Wood S (2009) The early pregnancy assessment project: the effect of cooperative care in the emergency department for management of early pregnancy complications. Aust N Z J Obstet Gynaecol 49:110-114. doi:10.1111/j.1479-828X.2009.00954.x

Potvin L, Lasker J, Toedter L (1989) Measuring grief: a short version of the perinatal grief scale. J Psychopathol Behav Assess 11:29-45

Rhone SA, Hodgson ZG, Moshrefzadeh A, Maurer C (2012) A Canadian urban early pregnancy assessment clinic: a review of the first year of operation. J Obstet Gynaecol Can 34:243-249. doi:10.1016/S17012163(16)35184-2

Rowlands IJ, Lee C (2010a) Adjustment after miscarriage: predicting positive mental health trajectories among young Australian women. Psychol Health Med 15:34-49. doi:10.1080/13548500903440239

Rowlands IJ, Lee C (2010b) 'The silence was deafening': social and health service support after miscarriage. J Reprod Infant Psychol 28:274-286. doi:10.1080/02646831003587346

Simmons RK, Singh G, Maconochie N, Doyle P, Green J (2006) Experience of miscarriage in the UK: qualitative findings from the National Women's Health Study. Soc Sci Med 63:1934-1946. doi: 10.1016/j.socscimed.2006.04.024

Toedter LJ, Lasker JN, Janssen HJEM (2001) International comparison of studies using the perinatal grief scale: a decade of research on pregnancy loss. Death Stud 25:205-228. doi:10.1080/074811801750073251

Tunde-Byass M, Cheung VYT (2009) The value of the early pregnancy assessment clinic in the management of early pregnancy complications. J Obstet Gynaecol Can 31:841-844. doi:10.1016/S17012163(16)34302-X

Warner A, Saxton A, Indig D, Fahy K, Horvat L (2012) Women's experience of early pregnancy care in the emergency department: a qualitative study. Australas Emerg Nurs J 12:86-92. doi:10.1016/j.aenj. 2012.02.002 